\title{
Spectrum of plant and animal diet of European Great Bustard (Otis tarda tarda) - an overview
}

\section{Sándor FARAGÓ}

Received: March 22, 2019 - Revised: April 04, 2019 - Accepted: April 05, 2019

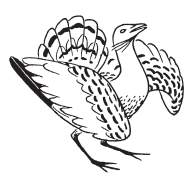

Faragó, S. 2019. Spectrum of plant and animal diet of European Great Bustard (Otis tarda tarda) - an overview. - Ornis Hungarica 27(1): 62-84. DOI: 10.2478/orhu-2019-0004

Abstract We have pointed out 272 plant and 217 animal, altogether 489 taxa in the diet of Great Bustard on the basis of data received from 9 (10) countries for Otis tarda tarda (Portugal, Spain, United Kingdom, Germany, Austria, Slovakia, Hungary, Ukraine, Kazakhstan, former Soviet Union). Out of 272 plant taxa, 40 were classified as cultivated plants, 232 wild plants and weeds. From the latter, 43 taxa were monocotyledons and 189 were dicotyledons. Animal food is shared among Annelida ( $\mathrm{n}=3)$, Arthropoda (189) Mollusca (2) and Vertebrata (23) phyla. Arthropods are mostly represented with Insecta (181), Arachnoidea (3), Chilopoda (2), Diplopoda (2) and Crustacea (mostly Isopoda) (1) classes. The component of the diet is possibly not related to selection but to the change of the abundance and availability of food and the ever present demand for animal food needed for the organism. Owing to the high number of taxa known as food, Great Bustard is definitely a generalist species. Due to the wide spectrum of animal taxa and because of the ability to subsidize the inefficient quality of food with quantity, Great Bustards can be regarded as a species with positive adaptation ability. It can be explained with a wide plant and animal food spectrum that Great Bustards even in intensive agricultural habitats can find food with indispensable quantity and quality.

Keywords: European Great Bustard, Otis tarda tarda, plant diet, animal diet, spectrum of diet

Összefoglalás Az Otis tarda tarda areájának 9 (10) országából (Portugália, Spanyolország, Egyesült Királyság, Németország, Ausztria, Szlovákia, Magyarország, Ukrajna, Kazahsztán, egykori Szovjetunió) származott közlések alapján a túzok táplálékaként 272 növényi és 217 állati, összesen 489 taxont mutattunk ki.

A 272 növényi taxon közül 40 termesztett növény, 232 vadon élö, illetve gyomnövény volt. Ez utóbbi közül 43 taxon egyszikủ és 189 taxon kétszikủ volt. Az állati táplálék az Annelida (3 taxon), Arthropoda (189) a Mollusca (2) és Vertebrata (23) törzsek között oszlott meg. Az Arthropodákat leginkább az Insecta (181), Arachnoidea (3), Chilopoda (2), Diplopoda (2), Crustacea (elsősorban Isopoda) (1) osztályok képviselik. A táplálék összetétele feltehetően nem a válogatással, hanem a táplálék bőségének, hozzáférhetőségének változásával, illetve a szervezet állati táplálék iránti mindenkori igényével függ össze. A táplálékként ismert taxonok magas száma okán a túzokot egyértelmüen generalista fajnak kell tekinteni. A fogyasztott növény és állati taxonok széles spektruma miatt, továbbá azon képessége alapján, hogy a táplálék elégtelen minőségét mennyiséggel tudja pótolni a túzok, a faj pozitív adaptációs képességének tekinthető.

A széles növényi és állati táplálékspektrummal magyarázható, hogy a túzok még az intenzív mezőgazdasági területeken is megtalálja a számára elengedhetetlen mennyiségü és minőségủ táplálékot.

Kulcsszavak: európai túzok, Otis tarda tarda, növényi táplálék, állati táplálék, táplálékspektrum

Institute of Wildlife Management and Vertebrate Zoology, University of Sopron, 9400 Sopron, Bajcsy-Zsilinszky u. 4., Hungary, e-mail: farago.sandor@uni-sopron.hu 


\section{Introduction}

During the conservation of the declining population (Alonso 2014) of the globally threatened Great Bustard (Otis tarda), it is inevitable to possess information on one of the most important segments of its feeding ecology, the trophic relations.

The former, general ornithological monographs (Glutz et al. 1973, Cramp \& Simmons 1980, Morales \& Martín 2002 etc.) address the diet of Great Bustard. In a previous work, Faragó (1986) detected 114 plant and 155 animal taxa as Great Bustard food on the distribution area of Otis tarda tarda, mostly based on bromathologic investigations collected by hunting. In many cases, the authors have provided only the family names of plants or animals, and the more precise classification was not possible as a result of the digestibility. Since our knowledge on this topic has increased, mostly because of the examination of faeces and many other new data found in specific literature resources, we have found it adequate to provide an overview on the bustard's diet spectrum.

\section{Material and methods}

The basis of the classification of the food list was given by the following publications, in which well-defined plant and animal taxa were shown for the Great Bustard. The publication years of these papers range 1781 and 2018. The study locations of these articles are as follows, listed by countries.

Portugal: Rocha et al. (2005)

Spain: Gil-Lletget (1945), Palaus Soler (1960), Palacios et al. (1975), Lucio (1985), Redondo \& Tortosa (1994), Hellmich (1995), Lane et al. (1999), Suárez (2002), Alonso \& Palacín (2009), Bravo et al. (2012), Bravo et al. (2014), Bravo et al. (2016)

United Kingdom: Jourdain (1948), Gooch et al. (2015)

Germany: Rörig (1900), Hennicke (1905), Niethammer (1942), Gewalt (1954), Gewalt (1959), Mansfeld (1958), Glutz et al. (1973), Heneberg (2016)

Austria: Dangel \& Winkler (1971), Rab et al. (2015)

Slovakia: Nečas \& Hanzl (1956)

Hungary: Huszthy (1781), Chernel (1899), Bodnár (1924), Greschik (1939), Vasvári (1949), Horváth (1958), Fodor et al. (1971), Sterbetz (1977), Faragó (1981), Faragó (1986), Faragó \& Csatári (1993), Faragó (2018)

Former Soviet Union: Dementiev \& Gladkov (1951), Spangenberg (1951), Stegman (1955), Isakov \& Flint (1989)

Ukraine: Spangenberg (1951), Kistjakinskij (1957)

Kazakhstan: Dolgushin (1962), Rjabov \& Ivanova (1971)

Since the Great Bustard is an omnivorous species, we separately discuss the plant and animal diet. Owing to the fact that in the taxonomy of plants and animals respectively, certain countries use different terminologies, and at the same time, we can find scientific synonyms 
in previous investigations, we also give both the scientific and the well-known synonyms as a compromise solution. However, we also give the source-publication as well in the case of each taxon.

In the case of the food list for plants, we declare the spectrum of the species in terms of cultivated plant, wild plants and weeds and then we give the family names - within that - in alphabetical order. In relation with plants, we use the Király (2009) nomenclature.

In the animal food list - due to its different particularity of taxonomy - we also share the ranging of phylum, classis, ordo, family, genus and species. Within orders (ordo) we give taxa in alphabetical order (including family, subfamily, genus and species). In relation with Coleoptera we have used the names of Merkl and Vig (2009).

\section{Result}

\section{Plant diet of Great Bustard}

\section{Cultivated plants consumed by Great Bustard}

Allium sativum - Spangenberg (1951), Cramp \& Simmons (1980)

Avena sativa - Gil-Lletget (1945), Mansfeld (1958), Fodor et al. (1971), Suárez (2002)

Beta vulgaris - Chernel (1899), Dangel \& Winkler (1971), Glutz et al. (1973), Cramp \& Simmons (1980)

Brassica napus - Chernel (1899), Rörig (1900), Nečas \& Hanzl (1956), Mansfeld (1958), Gewalt (1959), Fodor et al. (1971), Glutz et al. (1973), Sterbetz (1977), Cramp \& Simmons (1980), Isakov \& Flint (1989), Gooch et al. (2015), Raab et al. (2015)

Brassica juncea - Gooch et al. (2015)

Brassica nigra - Glutz et al. (1973), Lucio (1985), Bravo et al. (2016)

Brassica oleracea - Jourdain (1948), Mansfeld (1958), Glutz et al. (1973), Cramp \& Simmons (1980), Suárez (2002)

Brassica rapa - Jourdain (1948), Mansfeld (1958), Glutz et al. (1973), Cramp \& Simmons (1980)

Cicer arietinum - Palacios et al. (1975), Lucio (1985), Bravo et al. (2012), Bravo et al. (2016)

Citrullus lanatus - Hellmich (1995)

Cucurbita pepo - Gooch et al. (2015)

Eruca vesicaria - Cramp \& Simmons (1980), Lucio (1985), Morales \& Martín (2002), Bravo et al. (2016)

Fragaria ananassa - Gewalt (1954)

Helianthus annuus - Fodor et al. (1971), Sterbetz (1977)

Hordeum sativum - Stegman (1906), Mansfeld (1958), Fodor et al. (1971), Gooch et al. (2015)

Hordeum vulgare - Lucio (1985), Lane et al. (1999), Bravo et al. (2012), Bravo et al. (2016) Lens culinaris - Palacios et al. (1975), Lucio (1985) 
Lupinus angustifolia - Lane et al. (1999)

Medicago sativa - Mansfeld (1958), Fodor et al. (1971), Glutz et al. (1973), Palacios et al. (1975), Cramp \& Simmons (1980), Lucio (1985), Isakov \& Flint (1989), Lane et al. (1999), Bravo et al. (2012), Gooch et al. (2015)

Olea europaea - Palacios et al. (1975), Redondo \& Tortosa (1994), Suárez (2002), Bravo et al. (2012), Delibes et al. (2012), Bravo et al. (2016)

Onobrychis viciifolia - Mansfeld (1958), Fodor et al. (1971), Glutz et al. (1973), Cramp \& Simmons (1980)

Oryza sativa - Fodor et al. (1971)

Panicum miliaceum - Stegman (1906), Fodor et al. (1971)

Papaver somniferum - Mansfeld (1958), Fodor et al. (1971)

Phaseolus vulgaris - Stegman (1906)

Pisum sativum - Jourdain (1948), Nečas \& Hanzl (1956), Mansfeld (1958), Fodor et al. (1971), Glutz et al. (1973), Cramp \& Simmons (1980), Bravo et al. (2016)

Prunus domestica - Glutz et al. (1973)

Raphanus sativus - Mansfeld (1958), Glutz et al. (1973), Cramp \& Simmons (1980)

Ribes rubrum - Glutz et al. (1973)

Secale cereale - Mansfeld (1958), Fodor et al. (1971)

Sorgum sp. - Fodor et al. (1971)

Trifolium sp. - Jourdain (1948)

Trifolium pratense - Mansfeld (1958), Fodor et al. (1971), Glutz et al. (1973), Cramp \& Simmons (1980)

Trifolium repens - Mansfeld (1958), Fodor et al. (1971), Glutz et al. (1973), Cramp \& Simmons (1980)

Triticum sp. - Palacios et al. (1975)

Triticum aestivum - Chernel (1899), Bodnár (1924), Gil-Lletget (1945), Nečas \& Hanzl (1956), Mansfeld (1958), Fodor et al. (1971), Palacios et al. (1975), Sterbetz (1977), Lucio (1985), Lane et al. (1999), Gooch et al. (2015), Bravo et al. (2016)

Triticum turgidum - Suárez (2002)

Vicia sativa - Bodnár (1924), Fodor et al. (1971), Lucio (1985), Lane et al. (1999), Bravo et al. (2012), Bravo et al. (2016)

Vitis vinifera - Gil-Lletget (1945), Palacios et al. (1975), Lucio (1985), Lane et al. (1999), Suárez (2002), Bravo et al. (2012), Bravo et al. (2016)

Zea mays - Stegman (1906), Mansfeld (1958), Fodor et al. (1971)

\section{Wild plants and weeds consumed by Great Bustrad}

\section{Dicotyledonopsida}

Fagaceae

Quercus sp. - Glutz et al. (1973)

Quercus coccifera - Spangenberg (1951)

\section{Polygonaceae}

Polygonum sp. - Thaisz (1899) 
Polygonum aviculare - Lane et al. (1999), Bravo et al. (2012), Bravo et al. (2016)

Polygonum convolvulus - Kistjakinskij (1957)

Polygonum lapathifolium - Mansfeld (1958), Fodor et al. (1971)

Rumex pulcher - Bravo et al. (2012)

\section{Chenopodiaceae}

Atriplex hastata (prostrata) - Lucio (1985)

Chenopodium sp. - Bravo et al. (2012)

Chenopodium album - Lane et al. (1999), Gooch et al. (2015), Bravo et al. (2016)

Salsola kali - Palacios et al. (1975)

\section{Amaranthaceae}

Amaranthus sp. - Lucio (1985), Bravo et al. (2012)

\section{Caryophyllaceae}

Silenoideae - Palacios et al. (1975)

Cerastium holosteoides - Lane et al. (1999), Bravo et al. (2016)

Holosteum umbellatum - Lane et al. (1999), Bravo et al. (2016)

Sagina apetala - Lucio (1985)

Silene sp. - Lane et al. (1999)

cf. Spergula sp. - Palacios et al. (1975)

Spergula arvensis - Palacios et al. (1975)

Spergularia sp. - Lane et al. (1999)

Spergularia rubra - Lucio (1985), Suárez (2002)

Stellaria media - Mansfeld (1958), Fodor et al. (1971)

Vicia sp. - Suárez (2002)

\section{Ranunculaceae}

Ranunculus arvensis - Lucio (1985), Lane et al. (1999), Bravo et al. (2016)

Ranunculus repens - Lucio (1985)

\section{Papaveraceae}

Papaver roeas - Lane et al. (1999), Bravo et al. (2012), Bravo et al. (2016)

Roemeria hybrida - Bravo et al. (2016)

\section{Brassicaceae (Cruciferae)}

Brassiceae - Palacios et al. (1975)

Alyssum minus - Lane et al. (1999), Bravo et al. (2012), Bravo et al. (2016)

Biscutella auriculata - Bravo et al. (2012), Bravo et al. (2016)

Brassica sp. - Palacios et al. (1975), Lucio (1985), Suárez (2002)

Brassica barrelieri - Palacios et al. (1975)

Camelina sp. - Lane et al. (1999), Bravo et al. (2012)

Camelina microcarpa - Lane et al. (1999), Bravo et al. (2016)

Camelina sativa - Glutz et al. (1973), Lane et al. (1999)

Capsella bursa-pastoris - Mansfeld (1958), Fodor et al. (1971), Lucio (1985), Lane et al. (1999), Suárez (2002), Bravo et al. (2012), Gooch et al. (2015), Bravo et al. (2016)

Descurainia sophia - Bravo et al. (2012), Bravo et al. (2016)

Diplotaxis sp. - Cramp \& Simmons (1980)

Diplotaxis catolica - Palacios et al. (1975) 
Diplotaxis erucoides - Lane et al. (1999), Bravo et al. (2016)

Erophila verna - Lane et al. (1999), Suárez (2002)

Lepidium sp. - Lucio (1985)

Lepidium heterophyllum - Lucio (1985)

Malcolmia africana - Bravo et al. (2016)

Neslia paniculata - Bravo et al. (2016)

Rapistrum sp. - Bravo et al. (2016)

Raphanus raphanistrum - Rörig (1900), Mansfeld (1958), Glutz et al. (1973), Palacios et

al. (1975), Cramp \& Simmons (1980), Bravo et al. (2012), Bravo et al. (2016)

Sinapis arvensis - Chernel (1899), Bodnár (1924), Fodor et al. (1971)

Sisymbrium sp. - Bravo et al. (2012)

\section{Resedaceae}

Reseda lutea - Gooch et al. (2015)

\section{Rosaceae}

Potentilla anserina - Fodor et al. (1971)

Sanguisorba minor - Palacios et al. (1975), Lane et al. (1999)

\section{Fabaceae (Papilionaceae)}

Anthyllis vulneraria - Gooch et al. (2015)

Astragalus sp. - Lucio (1985), Bravo et al. (2012)

Astragalus incanus - Bravo et al. (2016)

Cicer sp. - Palacios et al. (1975)

Cicer arietinum - Suárez (2002)

Coronilla scorpioides - Lane et al. (1999), Bravo et al. (2016)

Lathyrus sp. - Lucio (1985), Bravo et al. (2016)

Lathyrus sativus - Gil-Lletget (1945)

Lens squlenta - Bravo et al. (2016)

Lotus sp. - Lucio (1985)

Medicago sp. - Palacios et al. (1975), Cramp \& Simmons (1980), Suárez (2002), Bravo et al. (2012), Bravo et al. (2016)

Medicago turbinata - Palacios et al. (1975)

Medicago minima - Palacios et al. (1975)

Medicago cf. polycarpa - Palacios et al. (1975)

Medicago polymorpha - Lucio (1985)

Melilotus sp. - Palacios et al. (1975)

Ononis sp. - Suárez (2002)

Ononis spinosa - Lucio (1985), Bravo et al. (2012), Bravo et al. (2016)

cf. Ornithopus sp. - Palacios et al. (1975)

Ornithopus compressus - Lane et al. (1999), Suárez (2002), Bravo et al. (2016)

Ornithopus sativus - Mansfeld (1958)

Scorpiurus sp. - Palacios et al. (1975)

Trifolium sp. - Palacios et al. (1975), Lane et al. (1999), Suárez (2002), Bravo et al. (2012), Gooch et al. (2015), Bravo et al. (2016)

Trifolium angustifolium - Lane et al. (1999), Bravo et al. (2016) 
Trigonella monspeliaca - Bravo et al. (2016)

Vicia sp. - Palacios et al. (1975), Bravo et al. (2012), Bravo et al. (2016)

Geraniaceae

Erodium sp. - Bravo et al. (2016)

Erodium cicutarium - Lane et al. (1999)

Geranium sp. - Bravo et al. (2012)

Geranium molle - Lane et al. (1999)

\section{Linaceae}

Linum sp. - Fodor et al. (1971)

\section{Euphorbiaceae}

Euphorbia serrata $\uparrow-$ Lucio (1985)

\section{Malvaceae}

Malva sylvestris - Bravo et al. (2012), Bravo et al. (2016)

\section{Onagraceae}

Epilobium sp. - Bravo et al. (2012)

\section{Apiaceae (Umbelliferae)}

Daucoideae - Palacios et al. (1975)

Caucalis sp. - Bodnár (1924)

Conium maculatum $\uparrow-$ Glutz et al. (1973), Cramp \& Simmons (1980)

Daucus sp. - Palacios et al. (1975), Suárez (2002)

Daucus carota - Lucio (1985), Lane et al. (1999)

Erygium (syn. Eryngium) sp. - Gil-Lletget (1945)

Pimpinella sp. - Chernel (1899)

Thapsia villosa - Lucio (1985)

Torilis nodosa - Bravo et al. (2016)

\section{Primulaceae}

Anagallis arvensis - Bravo et al. (2016)

Primula elatior - Gooch et al. (2015)

\section{Plumbaginaceae}

Limonium gmelini - Faragó \& Csatári (1993)

\section{Convolvulaceae}

Convolvulus sp. - Gil-Lletget (1945), Bravo et al. (2016)

Convolvulus arvensis - Chernel (1899), Palacios et al. (1975), Lucio (1985), Lane et al. (1999), Suárez (2002), Bravo et al. (2012)

\section{Boraginaceae}

Boraginaceae - Gooch et al. (2015)

Alkanna lutea - Lucio (1985)

Anhusa azurea - Bravo et al. (2016)

Echium sp. - Lucio (1985), Bravo et al. (2016)

Echium plantagineum - Lane et al. (1999), Bravo et al. (2016)

Heliotropium europaeum - Lane et al. (1999), Bravo et al. (2012), Bravo et al. (2016)

Lithospermum sp. - Bravo et al. (2016)

Myosotis sp. - Lucio (1985), Lane et al. (1999) 


\section{Lamiaceae (Labiatae)}

Lamium amplexicaule - Bravo et al. (2012), Bravo et al. (2016)

Salvia verbenacea - Bravo et al. (2016)

\section{Solanaceae}

Solanum nigrum - Lucio (1985), Lane et al. (1999), Bravo et al. (2012), Bravo et al. (2016)

\section{Scrophulariaceae}

Kickxia spuria - Bravo et al. (2012)

Veronica sp. - Gooch et al. (2015), Bravo et al. (2016)

Veronica hederifolia - Lucio (1985), Lane et al. (1999), Bravo et al. (2016)

Veronica triphyllos - Lane et al. (1999)

\section{Orobanchaceae}

Parentucellia latifoia - Lane et al. (1999)

\section{Rubiaceae}

Asperula cf. arvensis - Palacios et al. (1975)

Galium sp. - Suárez (2002)

Galium tricornutum - Bravo et al. (2012), Bravo et al. (2016)

Sherardia arvensis - Bravo et al. (2016)

\section{Plantaginaceae}

Plantago sp. - Jourdain (1948), Cramp \& Simmons (1980), Lane et al. (1999), Bravo et al. (2012), Bravo et al. (2016)

Plantago coronopus - Palacios et al. (1975), Lane et al. (1999)

Plantago lanceolata - Mansfeld (1958), Fodor et al. (1971), Palacios et al. (1975), Lucio (1985), Lane et al. (1999), Gooch et al. (2015)

Plantago major - Mansfeld (1958), Fodor et al. (1971)

Plantago media - Mansfeld (1958), Fodor et al. (1971)

\section{Valerianaceae}

Valerianella sp. - Hennicke (1905), Jourdain (1948)

\section{Dipsacaceae}

Cephalaria syriaca - Lane et al. (1999)

Scabiosa sp. - Lane et al. (1999)

Scabiosa stellata - Bravo et al. (2016)

\section{Campanulaceae}

Jasione montana - Suárez (2002)

\section{Asteraceae (Compositae)}

Achillea ageratum - Bravo et al. (2012)

Achillea millefolium - Mansfeld (1958), Fodor et al. (1971)

Anacyclus clavatus - Lane et al. (1999), Bravo et al. (2012), Bravo et al. (2016)

Andryala integrifolia - Lane et al. (1999), Bravo et al. (2012), Bravo et al. (2016)

Anthemis sp. - Palacios et al. (1975), Lucio (1985)

Anthemis arvensis - Lane et al. (1999)

Anthemis cotula - Palacios et al. (1975)

Apargia sp. - Jourdain (1948) 
Arnoseris sp. - Cramp \& Simmons (1980)

Bellis perennis - Lane et al. (1999)

Carduus tenuiflorus - Bravo et al. (2012)

Carthamus lanatus - Lane et al. (1999), Bravo et al. (2016)

Chondrilla juncea - Bravo et al. (2012)

Cichorium intybus - Bravo et al. (2012)

Cirsium sp. - Bravo et al. (2016)

Cirsium arvense - Nečas \& Hanzl (1956), Glutz et al. (1973), Cramp \& Simmons (1980), Lucio (1985)

Cnicus benedictus - Bravo et al. (2016)

Conyza canadensis - Bravo et al. (2012)

Corimbiferae - Palacios et al. (1975)

Crepis sp. - Jourdain (1948), Cramp \& Simmons (1980), Lucio (1985), Gooch et al. (2015)

Crepis virens (capillaris) - Lucio (1985)

Filago sp. - Bravo et al. (2016)

Filago pyramidata - Lane et al. (1999)

Galinsoga parviflora - Mansfeld (1958), Fodor et al. (1971)

Hedypnois cretica - Suárez (2002)

Hedypnois polymorpha - Palacios et al. (1975)

Hieracium sp. - Jourdain (1948), Cramp \& Simmons (1980), Gooch et al. (2015)

Hieracium aurantiacum - Gooch et al. (2015)

Hyoseris sp. - Jourdain (1948)

Hypochaeris (syn. Hypohoesris) sp. - Palacios et al. (1975), Cramp \& Simmons (1980)

Hypochaeris (syn. Hypohoeris) glabra - Palacios et al. (1975)

Lactuca serriola - Bravo et al. (2012)

Lactuca viminea - Lane et al. (1999)

Lapsana communis - Gooch et al. (2015)

Leontodon sp. - Jourdain (1948), Glutz et al. (1973), Cramp \& Simmons (1980)

Leontodon taraxacoides - Bravo et al. (2012)

Leontodon hispidus - Gooch et al. (2015)

cf. Leucanthemum - Palacios et al. (1975)

Leucanthemum vulgare - Gooch et al. (2015)

Mantisalca salmantica - Bravo et al. (2012)

cf. Podospermum sp. - Palacios et al. (1975), Cramp \& Simmons (1980)

Podospermum laciniatum - Palacios et al. (1975)

Scolymus sp. - Palacios et al. (1975)

Scorzonera sp. - Dolgushin (1962), Isakov \& Flint (1989), Bravo et al. (2016)

Senecio sp. - Palacios et al. (1975), Gooch et al. (2015)

Senecio vulgaris - Lane et al. (1999)

Sonchus sp. - Lucio (1985), Suárez (2002)

Sonchus arvensis - Lucio (1985)

Sonchus oleraceus - Glutz et al. (1973), Cramp \& Simmons (1980), Lucio (1985), Suárez (2002), Bravo et al. (2016) 
Tanacetum vulgare - Mansfeld (1958), Glutz et al. (1973), Cramp \& Simmons (1980) Taraxacum sp. - Palacios et al. (1975), Cramp \& Simmons (1980), Lucio (1985), Isakov \& Flint (1989), Lane et al. (1999)

Taraxacum officinale - Mansfeld (1958), Fodor et al. (1971), Glutz et al. (1973), Palacios et al. (1975), Lucio (1985), Bravo et al. (2012), Gooch et al. (2015), Bravo et al. (2016) Thrincia sp. - Palacios et al. (1975), Cramp \& Simmons (1980)

Thrincia hispida - Palacios et al. (1975)

Thrincia hirta - Palacios et al. (1975)

Tolpis (Hieracium) barbata - Lane et al. (1999), Bravo et al. (2016)

cf. Tragopogon sp. - Dolgushin (1962), Palacios et al. (1975), Isakov \& Flint (1989)

Tripleurospermum perforatum - Gooch et al. (2015)

Tubiflorae - Palacios et al. (1975)

\section{Monocotyledonopsida}

\section{Colchicaceae}

Muscari sp. - Bravo et al. (2016)

Muscari comosum - Lane et al. (1999)

Muscari racemosum (neglectum) - Palacios et al. (1975), Lane et al. (1999)

Ornithogalum umbellatum - Rörig (1900), Glutz et al. (1973), Cramp \& Simmons (1980)

\section{Alliaceae}

Allium sp. - Glutz et al. (1973)

cf. Allium longicuspis - Dementiev \& Gladkov (1951)

\section{Juncaceae}

Luzula sylvatica - Lucio (1985)

Poaceae (Gramineae) - Thaisz (1899), Sterbetz (1977), Cramp \& Simmons (1980), Faragó (1981), Faragó \& Csatári (1993), Bravo et al. (2012)

Aegilops sp. - Palacios et al. (1975), Bravo et al. (2016)

Aegilops ovata - Palacios et al. (1975)

Agropyron repens - Glutz et al. (1973), Cramp \& Simmons (1980)

Aira caryophyllea - Palacios et al. (1975)

Antinoria agrostidea - Lane et al. (1999)

Avena fatua - Lucio (1985)

Avena sterilis - Bravo et al. (2016)

Brachypodium pinnatum - Lucio (1985)

Bromus sp. - Fodor et al. (1971), Lucio (1985), Lane et al. (1999), Bravo et al. (2016)

Bromus diandrus - Lane et al. (1999)

Bromus rubens - Lane et al. (1999)

Bromus squarrosus - Bravo et al. (2016)

Bromus tectorum - Palacios et al. (1975)

Cynodon dactylon - Bravo et al. (2016)

Dactylis glomerata - Lucio (1985)

Echinochloa crus-galli - Fodor et al. (1971)

Festuca sp. - Sterbetz (1977), Isakov \& Flint (1989)

Festuca pseudovina - Fodor et al. (1971), Faragó \& Csatári (1993) 
Hordeum asperum - Lane et al. (1999)

Hordeum murinum - Fodor et al. (1971), Lane et al. (1999), Bravo et al. 2016

Lolium perenne - Lucio (1985)

Lolium rigidum - Bravo et al. (2016)

Mibora minima - Lane et al. (1999)

Phalaris sp. - Palacios et al. (1975), Lucio (1985)

cf. Phleum sp. - Palacios et al. (1975)

Phleum pratense - Lane et al. (1999)

Poa sp. - Palacios et al. (1975), Lucio (1985)

Poa annua - Lucio (1985), Lane et al. (1999)

Poa bulbosa - Palacios et al. (1975), Lane et al. (1999)

Poa pratensis - Fodor et al. (1971), Lucio (1985)

Setaria sp. - Bodnár (1924)

Taeniatherum caput-medusae - Bravo et al. (2016)

Tragus recemosus - Dolgushin (1962)

Vulpia sp. - Palacios et al. (1975), Lane et al. (1999)

Vulpia myuros - Palacios et al. (1975), Lucio (1985)

\section{Animal diet of Great Bustard}

\section{Annelida}

Oligochaeta - Rocha et al. (2005)

Lumbricidae - Jourdain (1948), Gewalt (1959), Cramp \& Simmons (1980)

Lumbricus terrestris - Fodor et al. (1971)

\section{Arthropoda}

\section{Crustacea}

Isopoda - Gewalt (1959), Cramp \& Simmons (1980)

Chilopoda - Rocha et al. (2005)

Scolopendra sp. - Palacios et al. (1975)

Diplopoda - Rocha et al. (2005)

Julus sp. - Suárez (2002)

\section{Insecta}

\section{Mantidea}

Mantidae - Rocha et al. (2005)

Mantis sp. - Spangenberg (1951), Rjabov \& Ivanova (1971), Bravo et al. (2012)

Mantis religiosa - Palacios et al. (1975), Suárez (2002)

\section{Orthoptera}

Acrididae - Spangenberg 1951, Dementiev \& Gladkov (1951), Dolgushin (1962), Rjabov \& Ivanova (1971), Palacios et al. (1975), Cramp \& Simmons (1980), Rocha et al. (2005), Bravo et al. (2014)

Acheta sp. - Palacios et al. (1975)

Calliptamus italicus - Palacios et al. (1975), Isakov \& Flint (1989)

Decticus verrucivorus - Gewalt (1959) 
Gryllidae - Dolgushin (1962), Cramp \& Simmons (1980), Rocha et al. (2005)

Gryllus campestris - Jourdain (1948), Spangenberg (1951), Gewalt (1959), Fodor et al. (1971), Sterbetz (1977)

Gryllotalpa gryllotalpa - Jourdain (1948), Spangenberg (1951), Gewalt (1959), Fodor et al. (1971), Palacios et al. (1975), Cramp \& Simmons (1980)

Oedipodinae - Palacios et al. (1975)

Pachytylus migratorius - Spangenberg (1951), Fodor et al. (1971)

Platystolus surcularius - Palacios et al. (1975)

Tettigonidae - Jourdain (1948), Dementiev \& Gladkov (1951), Rjabov \& Ivanova (1971), Cramp \& Simmons (1980), Isakov \& Flint (1989), Rocha et al. (2005)

Tettigonia (syn. Phasgonura) viridissima - Gewalt (1959), Palacios et al. (1975), Sterbetz (1977)

Dermaptera - Jourdain (1948), Cramp \& Simmons (1980)

Forficula sp. - Spangenberg (1951), Sterbetz (1977)

Forficula auricularia - Fodor et al. (1971)

Coleoptera - Dolgushin (1962)

Agriotes lineatus - Fodor et al. (1971)

Alleculidae - Bravo et al. (2016)

Amphimallon solstitialis - Gewalt (1959), Fodor et al. (1971), Sterbetz (1977)

Anisoplia sp. - Palacios et al. (1975)

Anisoplia austriaca - Kistjakinskij (1957)

Anomala sp. - Rörig (1900), Hennicke (1905)

Anomala vitis - Sterbetz (1977)

Apion sp. - Mansfeld (1958)

Aromia sp. - Jourdain (1948)

Aromia moschata - Hennicke (1905)

Asida sp. - Palacios et al. (1975), Lucio (1985)

Asidae - Palacios et al. (1975)

Blittophaga opaca - Mansfeld (1958)

Blittophaga undata - Rörig (1900), Mansfeld (1958), Gewalt (1959)

Brachycerus barbarus - Palacios et al. (1975), Suárez (2002)

Brachyderinae - Palacios et al. (1975)

Buprestidae - Rocha et al. (2005)

Calandrynae - Palacios et al. (1975)

Calosoma sp. - Jourdain (1948)

Calosoma sycophanta - Hennicke (1905)

Calosoma denticolla - Kistjakinskij (1957)

Cantharidae - Bravo et al. (2016)

Cantharis sp. - Suárez (2002)

Capnodis tenebricosa - Palacios et al. (1975)

Carabidae - Nečas \& Hanzl (1956), Kistjakinskij (1957), Dolgushin (1962), Rjabov \& Ivanova (1971), Palacios et al. (1975), Cramp \& Simmons (1980), Isakov \& Flint (1989), Lane et al. (1999), Rocha et al. (2005), Bravo et al. (2012), Bravo et al. (2016) 
Carabus sp. - Rörig (1900), Hennicke (1905), Fodor et al. (1971)

Carabus auratus - Niethammer (1942), Gewalt (1959), Glutz et al. (1973)

Carabus nitens - Niethammer (1942), Gewalt (1959)

Cassida sp. - Jourdain (1948), Lucio (1985)

Cassida nebulosa - Rörig (1900), Hennicke (1905), Gewalt (1959)

Cassida subferruginea - Kistjakinskij (1957)

Cerambicidae - Dolgushin (1962), Rocha et al. (2005), Bravo et al. (2016)

Cetonia aurata - Huszthy (1781), Nečas \& Hanzl (1956), Dolgushin (1962)

Chrysomela sp. - Lucio (1985)

Chrysomela banksi - Suárez (2002)

Chrysomela fastuosa - Gewalt (1959)

Chrysomela polita - Gewalt (1959)

Chrysomela sanguinolenta - Rörig (1900)

Chrysomelidae - Dolgushin (1962), Cramp \& Simmons (1980), Lucio (1985), Lane et al. (1999), Rocha et al. (2005), Bravo et al. (2012), Bravo et al. (2016)

Cleonus sp. - Rörig (1900), Hennicke (1905), Bodnár (1924), Jourdain (1948)

Cleonus nigrivittis - Kistjakinskij (1957)

Cleonus piger - Glutz et al. (1973)

Cleonus punctiventris - Kistjakinskij (1957)

Coccinella septempunctata - Palacios et al. (1975), Lucio (1985), Suárez (2002)

Coccinellidae - Lane et al. (1999)

Coniocleonus sp. - Palacios et al. (1975), Suárez (2002)

Cryptocephalus sp. - Lucio (1985)

Cryptocephalus sericeus - Gewalt (1959)

Curculionidae - Hennicke (1905), Nečas \& Hanzl (1956), Dolgushin (1962), Rjabov \& Ivanova (1971), Palacios et al. (1975), Cramp \& Simmons (1980), Lucio (1985) Isakov \& Flint (1989), Lane et al. (1999), Rocha et al. (2005), Bravo et al. (2012), Bravo et al. (2014), Bravo et al. (2016)

Cycloderes sp. - Palacios et al. (1975), Suárez (2002)

Dorcadion aethiops - Fodor et al. (1971), Sterbetz (1977)

Dorcadion fulvum - Nečas \& Hanzl (1956), Fodor et al. (1971), Sterbetz (1977)

Dytiscus sp. - Jourdain (1948)

Elater sp. - Jourdain (1948)

Elateridae - Chernel (1899), Hennicke (1905), Mansfeld (1958), Gewalt (1959), Palacios et al. (1975)

Epuraea sp. - Lucio (1985)

Erodiinae - Palacios et al. (1975)

Eusomus sp. - Bodnár (1924)

Geotrupes sp. - Rörig (1900), Hennicke (1905), Jourdain (1948), Palacios et al. (1975)

Geotrupes cf. stercorarius - Palacios et al. (1975)

Geotrupes laevigatus - Suárez (2002)

Geotrupinae - Dolgushin (1962), Isakov \& Flint (1989)

Haltica sp. - Jourdain (1948) 
Haltica oleracea - Hennicke (1905)

Hellopatus sp. - Lucio (1985)

Helopinae - Palacios et al. (1975)

Histeridae - Lane et al. (1999)

Hister fimetarius - Glutz et al. (1973)

Hypera sp. - Palacios et al. (1975), Suárez (2002)

Hylobiinae - Palacios et al. (1975)

cf. Labidostomis sp. - Palacios et al. (1975)

Lachnaea sexpunctata - Palacios et al. (1975)

Larinus sp. - Palacios et al. (1975)

Larinus buccinator - Suárez (2002)

Leptinotarsa decemlineata - Gewalt (1959), Fodor et al. (1971), Glutz et al. (1973), Sterbetz (1977), Cramp \& Simmons (1980)

Leucosomus pedestris - Nečas \& Hanzl (1956)

Meloë sp. - Vasvári (1942), Nečas \& Hanzl (1956), Kistjakinskij (1957), Palaus Soler (1960), Dolgushin (1962), Palacios et al. (1975), Lucio (1985)

Meloë collaris - Lucio (1985)

Meloë (syn. Physomeloe) corallifer - Palacios et al. (1975), Lucio (1985)

Meloë hungarus - Greschik (1939)

Meloë (syn. Berberomeloe) majalis - Entz (1904), Palacios et al. (1975), Lucio (1985), Suárez (2002), Sánchez-Barbudo et al. (2012)

Meloë proscarabeus - Vasvári (1942), Palacios et al. (1975)

Meloë rugosus - Vasvári (1942)

Meloë tuccius - Palacios et al. (1975), Suárez (2002)

Meloë variegatus - Vasvári (1942), Lucio (1985)

Meloë violaceus - Greschik (1939), Vasvári (1942), Lucio (1985)

Meloë violaclur - Vasvári (1942)

Meloidae - Palacios et al. (1975), Lane et al. (1999), Rocha et al. (2005), Bravo et al. (2014), Bravo et al. (2016), Heneberg (2016)

Melolontha sp. - Jourdain (1948)

Melolontha melolontha - Nečas \& Hanzl (1956), Mansfeld (1958), Fodor et al. (1971), Glutz et al. (1973), Sterbetz (1977)

Melolonthinae - Isakov \& Flint (1989)

Melyridae - Lane et al. (1999), Rocha et al. (2005)

Microlarinus sp. - Suárez (2002)

Micrositus sp. - Lucio (1985)

Mylabris quadripunctata - Kistjakinskij (1957)

Nitidulidae - Rocha et al. (2005)

Opatrum sabulosum - Fodor et al. (1971), Sterbetz (1977)

Otiorrhynchus sp. - Rörig (1900), Hennicke (1905), Bodnár (1924), Jourdain (1948)

Oxythyrea funesta - Palacios et al. (1975)

Phalacridae - Rocha et al. (2005)

Phyliam sp. - Lucio (1985) 
Phyliam abreviatus. - Lucio (1985)

Phytodecta formicata - Fodor et al. (1971)

Phytodecta variabilis - Suárez (2002)

Phytonomus variabilis - Fodor et al. (1971)

Pimelia sp. - Palaus Soler (1960), Palacios et al. (1975), Suárez (2002)

Pimelia rugulosa - Palacios et al. (1975)

Pimelia baetiva - Lucio (1985)

Pimelia punctata - Lucio (1985)

Psilothrix cyaneus - Palacios et al. (1975)

Rhizortogus aequinoctialis - Fodor et al. (1971)

Rhynchiitinae - Palacios et al. (1975)

Rhytidoderes sp. - Palacios et al. (1975)

Scarabaeidae - Cramp \& Simmons (1980), Lucio (1985), Lane et al. (1999), Rocha et al. (2005), Bravo et al. (2012), Bravo et al. (2014), Bravo et al. (2016)

Scarabaeus sp. - Fodor et al. (1971), Sterbetz (1977)

Sepidium bidentatum - Palacios et al. (1975), Lucio (1985)

Silpha sp. - Jourdain (1948), Suárez (2002)

Silpha atracta - Hennicke (1905)

Silpha obscura - Gewalt (1959)

Silpha reticulata - Hennicke (1905)

Silphidae - Cramp \& Simmons (1980)

Staphylinidae - Palacios et al. (1975), Lane et al. (1999), Rocha et al. (2005)

Staphylinus sp. - Suárez (2002)

Stenus sp. - Suárez (2002)

Subcoccinella vigintiquatuorpunctata - Fodor et al. (1971)

Tenebrionidae - Dolgushin (1962), Rjabov \& Ivanova (1971), Palacios et al. (1975), Cramp \& Simmons (1980), Lucio (1985), Isakov \& Flint (1989), Lane et al. (1999), Rocha et al. (2005), Bravo et al. (2012), Bravo et al. (2012), Bravo et al. (2014), Bravo et al. (2016)

Tentyria sp. - Suárez (2002)

Tentyria bassil - Lucio (1985)

Timarcha sp. - Suárez (2002)

Tropinota hirta - Palacios et al. (1975), Lucio (1985)

Tropinota squalida - Suárez (2002)

Zabrus tenebrioides - Fodor et al. (1971), Sterbetz (1977)

Zonabris (Mylabris) sp. - Palacios et al. (1975)

\section{Hymenoptera}

Formica sp. - Palacios et al. (1975)

Formicidae - Dementiev \& Gladkov (1951), Fodor et al. (1971), Isakov \& Flint (1989), Rocha et al. (2005), Bravo et al. (2012), Bravo et al. (2014), Bravo et al. (2016) cf. Braconidae - Palacios et al. (1975)

Diptera - Jourdain (1948), Bravo et al. (2012), Bravo et al. (2016) 
Brachycera - Palacios et al. (1975)

Musca sp. - Gewalt (1959)

Lepidoptera - Rjabov \& Ivanova (1971), Palacios et al. (1975), Cramp \& Simmons (1980), Rocha et al. (2005), Bravo et al. (2012), Bravo et al. (2016)

Agrestis sp. - Bodnár (1924)

Cuculliinae - Palacios et al. (1975)

Hyles (syn. Deilephila, Celerio) euphorbiae - Gewalt (1959)

Hamestra sp. - Bodnár (1924)

Noctuidae - Gewalt (1959), Palacios et al. (1975)

Noctuinae - Palacios et al. (1975)

Papilio machaon - Gewalt (1959)

Pieridae - Gewalt (1959), Palacios et al. (1975)

Pieris brassicae - Gewalt (1959)

Hemiptera - Bravo et al. (2014)

Heteroptera - Hennicke (1905), Niethammer (1942), Gewalt (1959)

Carpocoris fuscispinus - Suárez (2002)

Eurydema sp. - Fodor et al. (1971), Sterbetz (1977), Lucio (1985)

Eurydema ornatum - Suárez (2002)

Eurygaster sp. - Fodor et al. (1971), Sterbetz (1977), Lucio (1985)

Eurygaster austriaca - Suárez (2002)

Eurygaster maura - Kistjakinskij (1957), Suárez (2002)

Nabis sp. - Suárez (2002)

Pentatomidae - Palacios et al. (1975), Bravo et al. (2016)

Reduvius personatus - Suárez (2002)

Scutellaridae - Glutz et al. (1973)

Stolia sp. - Suárez (2002)

Homoptera - Isakov \& Flint (1989)

Arachnoidea

Areaneidea - Jourdain (1948), Gewalt (1959), Dolgushin (1962), Palacios et al. (1975), Bravo et al. (2012), Bravo et al. (2016)

Araneae - Rocha et al. (2005)

Solifuga - Rocha et al. (2005)

Mollusca

\section{Gastropoda}

Agriolimax agrestis - Jourdain (1948), Fodor et al. (1971), Cramp \& Simmons (1980) Helix pomatia - Fodor et al. (1971), Cramp \& Simmons (1980)

\section{Vertebrata}

Amphibia - Jourdain (1948), Cramp \& Simmons (1980)

Hyla arborea - Gewalt (1954), Rjabov \& Ivanova (1971)

Bufo viridis - Rjabov \& Ivanova (1971)

Rana arvalis - Gewalt (1954)

Reptilia - Jourdain (1948), Dementiev \& Gladkov (1951), Dolgushin (1962), Cramp \& Simmons (1980) 
Lacerta sp. - Spangenberg (1951), Nečas \& Hanzl (1956)

Lacerta agilis - Rjabov \& Ivanova (1971)

Podarcis hispanicus - Gil-Lletget (1945)

Aves - Spangenberg (1951), Fodor et al. (1971)

Alauda arvensis - Chernel (1899), Jourdain (1948), Cramp \& Simmons (1980)

Alaudidae juv. - Dolgushin (1962)

Melanocoripha yeltonensis juv. - Dementiev \& Gladkov (1951), Spangenberg (1951)

Motacilla flava juv. - Rjabov \& Ivanova (1971)

Numenius arquata ov. - Gewalt (1954), Cramp \& Simmons (1980)

Oenanthe oenanthe juv. - Rjabov \& Ivanova (1971)

\section{Mammalia}

Lagurus lagurus - Glutz et al. (1973)

Lepus europaeus (juv.) - Jourdain (1948), Fodor et al. (1971), Cramp \& Simmons (1980)

Microtus arvalis - Chernel (1899), Gewalt (1959), Cramp \& Simmons (1980)

Microtus agrestis - Jourdain (1948)

Microtus (syn. Pitymys) duodecimcostatus - Palacios et al. (1975)

Microtus socialis - Dolgushin (1962)

Muridae - Dolgushin (1962), Rjabov \& Ivanova (1971), Sterbetz (1977)

Mus sp. - Fodor et al. (1971)

\section{Other ingredients in the stomach of Great Bustard}

For digestion, the Great Bustard swallows pebbles and quartz pieces so-called gastrolits even as chicks. Besides, Hennicke (1905) mentioned coins, Gewalt (1959) mentioned metal pieces and relatively large pieces of rubber. According to Nečas and Hanzl (1956), some other indigestible objects such as glass splinters, pieces of china also occur in the Great Bustard's stomach regularly. According to Moltoni (1968) in the county of Vicenza, Italy, the stomach of a shot-down young male Great Bustard also included a piece of ceramics, and a strongly corroded German 2 Pfennig coin. Sterbetz (1977) found gastrolits in the stomach of three bustards out of 16 . In one of these, there was a tile fragment with three $\mathrm{cm}$ diameter, in the other there were 2 pebbles of 6 and $10 \mathrm{~mm}$ and in the last one a pebble of $30 \mathrm{~mm}$ showed up. We conclude that the gastrolits assist in digestion by the fragmentation of the food.

\section{Discussion}

We have detected 272 plant and 217 animal, altogether 489 taxa as Great Bustard diet based on data received from 9 (10) countries for Otis tarda tarda area: (Portugal, Spain, United Kingdom, Germany, Austria, Slovakia, Hungary, Ukraine, Kazakhstan, former Soviet Union). Out of 272 plant taxa, there were 40 cultivated plants, 232 wild plants and weeds. From the latter, 43 taxa were monocotyledons and 189 were dicotyledons.

The families of wild plants and weeds taken, in succession were as follows: dicotyledons (30 families) - Asteraceae/Compositae (59 taxons), Fabaceae/Papillionaceae (26), Brassicaceae 
(22), Cariophyllacae (11), Apiaceae/Umbelliferae (9), Boraginaceae (8), Poligonaceae (5), Plantaginaceae (5), Chenopodiaceae (4), Geraniaceae (4), Scrophulariaceae (4), Rubiaceae (4), Dipsacaceae (3), Ranunculaceae (2), Papaveraceae (2), Rosaceae (2), Primulaceae (2), Convolvulacae (2), Lamiaceae/Labiatae (2), Fagaceae (2), Amaranthaceae (1), Resedaceae (1), Linaceae (1), Euphorbiaceae (1), Malvaceae (1), Onagraceae (1), Plumbaginaceae (1), Solanaceae (1), Orobanchaceae (1), Valerianaceae (1), Campanulaceae (1). Monocotyledons (4 families) - Poaceae/Gramineae (36 taxons), Colchicaceae (4), Alliaceae (2), Juncaceae (1).

Animal food is shared among Annelida (3 taxa), Arthropoda (189) Mollusca (2) and Vertebrata (23) phyla. Arthropods are mostly represented with Insecta (181), Arachnoidea (3), Chilopoda (2), Diplopoda (2), and Crustacea (mostly Isopoda) (1) classes.

The orders of Insects based on the regularity of taxon-numbers are as follows: Coleoptera (134), Orthoptera (12), Lepidoptera (10), Hemiptera (13), Mantidea (3), Dermaptera (3), Hymenoptera (3), Diptera (2), Homoptera (1).

The phylum of Vertebrates is represented by all the four terrestrial classes: Mammalia ( 8 taxa), Aves (7), Amphibia and Reptilia (4-4).

Considering the high number of 489 taxa taken as food, we definitely need to regard Great Bustard as a generalist species. The Bustard's great adaptation ability is the base and evidence of the wide spectrum of the consumed plant and animal taxa.

The cited, detailed publications also have shown that the volume of certain taxa in total, seasonally and also in various age-groups were different. This means that the inner rates of diet components constantly change.

Young Great Bustard chicks, but even older ones, consume fresh shoots, crops and seeds of plants. During the period of reproduction of female Great Bustards, ,grazing” cannot be observed, which in contrast, is a charasteristic type of behaviour in males. However, they consume more insects. During mating season, male Great Bustards feed on animals in a large proportion. Hens can very often digest rough plant parts and seeds. They defecate them even without digestion (Gewalt 1959).

In Spain, according to the examinations of Palacios et al. (1975), 90.2\% of the volume of spring was plant food. Most of plant diet was represented by the families of Compositae/ Asteraceae (51.3\%), a Fabaceae (11.4\%), a Cruciferae/Brassicaceae (11.4\%) and Gramineae/Poaceae (9.3\%). In diet composition made up by Arthropoda, coleopterans (Scarabaeidae, Curculionidae, Tenebrionidae and from the Meloidae family) dominated by $95.50 \%$. In the summer diet, the importance of green plant parts was decreasing, however, seeds of grains and Arthropods increased (Mantidae, Orthoptera and Formicidae). In the autumn aspect, comparing the summer one, there was no significant difference, but the consumption of cultivated plants was increasing. The rate of Orthoptera in the diet remained unchanged at the same time (Locustidae, Gryllidae), just like in the case of Hymenoptera (Formicidae). In the winter period, similarly to that of spring, the green plant parts dominated, though the animal diet was practically missing.

Based on the investigation of stomach content and summer/winter faeces, Lucio (1985) analysed the diet of Great Bustard, collected at the Duero Basin. During the whole year, alfalfa played an important role, though at the end of summer and in winter the seeds of winter wheat and winter barley, grapes and Papilionaceae dominated the diet. Besides cultivated 
plants, he detected the presence of 35 species belonging to the following families: Compositae/Asteraceae (8 species), Gramineae/Poaceae (8 species), Cruciferae/ Brassicaceae (6 species), Boraginaceae (3 species), Caryophyllaceae (2), Ranunculaceae (2), Plantaginaceae (1), Euphorbiaceae (1), Scrophulariaceae (1), Umbelliferae (2) és Juncaceae (1). In the spring season, most of the animal food in the investigated stomachs was Coleoptera $(97.16 \%)$ - within that mostly Tenebrionidae, Meloidae, Chrysomelidae - and Heteroptera. Out of the faeces collected during the summer period, coleopteras, hymenopteras and heteropteras were shown to be present with $20-47 \%$ frequency, though in winter Arthropoda was only possible to make out in one out of ten faeces.

The diet spectrum of a Northwest Spanish Great Bustard population was analysed based on faeces investigation by Lane et al. (1999). The analysis considering the data of the whole year, detected 65 plant species - some of them lacking in earlier analyses - in the course of consumption of summer, winter and autumn. The ratio of green plant parts referring to dry material was $48.4 \%$, and seeds in August 10.6\%. In the second half of summer, there were seeds of winter wheat and winter barley in the faeces. In the course of the whole year, alfalfa was the most chosen food component and types of grass were preferred to a lesser extent only. Besides plant food, the specimens of 8 Insect orders were found in the faeces, out of which Coleoptera, Hymenoptera and Orthoptera were available in the largest number and ratio. They consumed coleopteras during the whole year although ortopteras were eaten, in a smaller amount than they were disposable. Hymenopteras in May were of less significant, they were consumed mostly in September and November.

Based on the investigation of stomach content of young bustards, Bravo et al. (2012) concluded, that diet - referring to dry material - was 33\% arthropods, $30 \%$ green plant material and $23 \%$ seed. Gastrolits in stomachs were only be found in summer and autumn. Food components changed by aspects. In summer, they consumed mostly arthropods (50\%), green plant parts mostly in wintertime (56\%). The volume and the average size of the $\mathrm{Ar}$ thropod component - in the case of males - were larger than that of females, but there was no significant difference between sexes. In winter, weeds, Papilionaceae, seeds of cultivated plants dominated, although grain types were preferrably consumed and the seeds of these (wheat, barley) were of great importance during autumn and winter.

Based on faeces samples of 299 hens and 320 cocks, Bravo et al. (2016) investigated if there was a sex-specific difference between the food spectrum, diversity and the degree of overlaps of food spectra and size of Arthropod preys, working at 9 Spanish Great Bustard sites. They confirmed that both sexes were mainly herbivorous and they particularly consumed papilionaceous plants, if available. Males fed on less Arthropod diet than females, but at the same time, the size of those are significantly larger than in the case of females. The diet of males shows a bigger diversity than that of the hens, though except for the period after mating. The overlap in food between sexes was found to $b 0.7$, which is one of the smallest rates in the case of birds. The investigation has shown that in relation with the sexual dimorphism of particular scales, the difference between the sexes' dietary niche can be explained by the dissimilar reproduction role of Great Bustard males and females.

In southwest England, Gooch et al. (2015) examined the diet of the reintroduced Great Bustards on free territories, both in the time of without feeding and in the time of supplementary 
feeding (October-December). In the course of the investigation of the faeces sample, they used the method of microhystology. The ingredients of the food were formed in the function of plant availability and the period of phenology. Animals of lower orders were rarely made evident as food. The main food were green part and seeds of cultivated plants such as rape, mustard, barley, at the same time the monotocyledonous and dicotyledonous weeds were only of second importance (25\%).

Benchmark investigations were made in Kazakhstan (Rjabov \& Ivanova 1971) - adult (n $=25)$ and juveniles $(\mathrm{n}=12)$ birds - analysing stomach content. Among the listed taxa, there were 25 plant species; considering one stomach, it was usually dominated by $2-3$ species and 25 specimens, on average.

The great value of the investigation is that it provided the relations of volume as well. Accordingly, in the case of adult birds the relation of animal and plant food was found to be $37.8: 62.2$ volume $\%$, though in the case of chicks it is $96.5: 3.5$ volume $\%$. They have shown that from May to August, Great Bustards shifted from the dominance of plant materials to insect food, gradually. One of the reasons of this is that in nature, the availability of insects is increasing and that of plant decreasing, however, before migration (on the investigated area the Great Bustard is a regular migratory bird) the organism is required to accumulate protein and fat. At the same time, there is an interesting statement by declaring that with the increase of nutritional value, the fullness rate of the stomach was decreasing. The Great Bustard's ability to subsidize the inefficient quality of food with quantity is regarded to be positive adaptation ability by the authors. When the bird consumes food of low energy value (plants) it consumes a large quantity of that. From food of big nutritional value they consume only moderately. The mixed food makes it possible for Great Bustards to turn from food of one type into another one and they are capable of doing so quite quickly. The animal food for Great Bustards contains protein of $13-30 \%$, though in the case of plants it is $3.5-5.3 \%$. By aging, protein consumption for the body volume is decreasing; at the end of growing protein consumption nearly stops and in the case of adult specimens nitrogen-balance comes into place. It might be observed that out of the feeding birds with different ages, younger ones rather have animal, older ones plant food. The diversified component of these diet guarantees all the amino-acids needed for protein-synthesis.

To summarize, we can declare that in the food of young Great Bustards animal food, in the older ones plant food is dominating. The component of diet is possibly not related to selection, rather to the abundance of it and the change of availability recepectively to the ever present demand for animal food.

Simultaneously, it is important to concluce that it can de explained with a wide plant and animal food spectrum (richness) that Great Bustards even in intensive agricultural habitats can find food with indispensable quantity and quality. This is improved by the fact that neither in Europe (Kollar 1996, Nagy 2009, Alonso 2014) nor regional (Kollar 2001, Faragó 2004, Bankovics 2005, Alonso \& Palacín 2009, Spakovszky et al. 2011, Raab et al. 2014, Vadász \& Lóránt 2014, Faragó 2018) level can we find the lack of food among the endangaring factors for the globally threatened species as opposed e.g. to Grey Partridge (Perdix perdix) for instance (Potts 1986, 2012). 


\section{Request}

Neither the resource list nor the diet list gained from it can be complete, so the author wishes to be informed about references concerning missing data.

\section{References}

Alonso, J. C. 2014. The Great Bustard: past, present and future of a globally threatened species. - Ornis Hungarica 22 (2): 1-13. DOI: 10.2478/orhu-2014-0014

Alonso, J. C. \& Palacín, C. 2009. Avutarda - Otis tarda [The Great Bustard - Otis tarda]. - In: Salvador, A. \& Bautista, L. M. (eds.) Enciclopedia Virtual de los Vertebrados Españoles. - Museo Nacional de Ciencias Naturales, Madrid (in Spanish)

Bankovics, A. 2005. A general overview of the threats of Hungarian Great Bustards (Otis tarda). - Aquila 112: 135142.

Bodnár, B. 1924. A túzok táplálkozásáról. - Über die Nahrung von Otis tarda [About the alimentation of Great Bustard]. - Aquila 30-31: 308., 330-331. (in Hungarian and German)

Bravo, C., Ponce, C., Palacín, C. \& Alonso, J. C. 2012. Diet of young Great Bustard Otis tarda in Spain: sexual and seasonal differences. - Bird Study 59(2): 243-251. DOI: 10.1080/00063657.2012.662940

Bravo, C., Bautista, L. M., García-París, M., Blanco, G. \& Alonso, J. C. 2014. Males of a strongly polygynous species consume more poisonous food than females. - PLoS ONE 9: e111057. DOI: 10.1371/journal.pone.0111057

Bravo, C., Ponce, C., Bautista, L. M. \& Alonso, J. C. 2016. Dietary divergence in the most sexually size-dimorphic bird. - The Auk 133(2): 178-197. DOI: 10.1642/AUK-15-206.1

Chernel, I. 1899. A túzok Otis tarda L. 1758. [Great Bustard Otis tarda L. 1758.]. - In: Chernel, I. (ed.) Magyarország madarai, különös tekintettel gazdasági jelentőségökre [Birds of Hungary, with especial regard to their economic importance]. - Budapest. Második könyv. Tüzetes rész, pp. 227-231. (in Hungarian)

Cramp, S. \& Simmons, K. E. L. 1980. (eds.) Handbook of the birds of Europe, the Middle East and North Africa, Vol. 2. Hawks to bustards. - Oxford University Press, Oxford, pp. 636-668.

Dangel, M. \& Winkler, H. 1971. Interessante Todesursache freilebender Großtrappen [Interesting cause of death of free-living Great Bustard]. - Egretta 14 (1): 26. (in German)

Delibes, M., Corbacho, C., Calvo, G. \& Fedriani, J. M. 2012. Agriculture as matchmaker of an unexpected mutualism: Great Bustard disperses and enhances emergence of domestic olive seeds. - Basic and Applied Ecology 13(2): 125-131. DOI: 10.1016/j.baae.2011.11.003

Dementiev, G. P. \& Gladkov, N. A. 1951. Drofa - Otis tarda - Ptici Sovietskogo Sojuza, Vol. II. [Birds of Soviet Union, Vol. II.]. - Moscow, pp. 157-168. (in Russian)

Dolgushin, I. A. 1962. Ptici Kazakhstana, Vol. II. [Birds of Kazakhstan, Vol. II.]. - Izadatel'stvo Akademii Nauk Kazahskoj SSR, Institut Zoologi Alma-Ata, pp. 26-39. (in Russian)

Entz, G. 1904. Az állatok színe és a mimicry [The color of animals and the mimicry]. - Természettudományi Közlöny 36 (420): 465-486. (in Hungarian)

Faragó, S. 1981. Villanyvezeték okozta túzokpusztulások a Hanságban [Destructions of Great Bustards by electric power lines in Hanság]. - Madártani Tájékoztató 1981. júl-szept.: 136-137. (in Hungarian)

Faragó, S. 1986. Az európai túzok (Otis tarda Linné, 1758) növényi és állati eredetủ táplálékának fajspektruma az area területén [Spectrum of plant and animal diet of European Great Bustard (Otis tarda Linné, 1758) in the area]. - Erdészeti és Faipari Tudományos Közlemények 1985 (1-2): 121-130. (in Hungarian with German and English Summary)

Faragó, S. (ed.) 2004. Species Action Plans - Great Bustard (Otis tarda). - Office for Nature Conservation, Ministry of Environment and Water, Budapest

Faragó, S. 2018. A túzok a Kisalföldön [The Great Bustard in the Little Hungarian Plane]. - Sopron University Press, Sopron (in Hungarian)

Faragó, S. \& Csatári, G. 1993. Možnosti prevencie proti upchatiu žalúdka dropa vel'kého rastlinnou hmotou z potravy [Possibilities for the prevention of stomach congestion of the Great Bustard by plant substance from food]. Folia Venatoria 23: 201-207 (in Slovakian with English Summary)

Faragó, S., Spakovszky, P. \& Raab, R. 2014. Conservation of Great Bustard (Otis tarda) population of the Mosoni-Plain - A success story. - Ornis Hungarica 22: 14-31. DOI: 10.2478/orhu-2014-0015 
Fodor, T., Nagy, L. \& Sterbetz, I. 1971. A túzok [The Great Bustard]. - Mezőgazdasági Kiadó, Budapest (in Hungarian with German Summary)

Gewalt, W. 1954. Die Großen Trappen. - Europas Riesenvögel in der Mark [The Great Bustard - Europe's giant birds in the country]. - Berlin (in German)

Gewalt, W. 1959. Die Grosstrappe (Otis tarda L.) [The Great Bustard]. - A. Ziemsen Verlag, Wittenberg-Lutherstadt (in German)

Gil-Lletget, A. 1945. Bases para un estudio cientifico de alimentación de las aves y resultado del analosis de 400 estómagos [Basis for a scientific study of the birds and results of the analysis of 400 stomachs]. - Boletín de la Real Sociedad Española de Historia Natural 43(1-2): 9-23. (in Spanish)

Glutz, U., Bauer, K. \& Bezzel, E. 1973. Otis tarda Linné 1758 - Grosstrappe [The Great Bustard]. - Handbuch der Vögel Mitteleuropas. Bd. 5. Galliformes - Gruiformes. - AULA-Verlag GmbH, Wiesbaden. 2. durchgesehene Auflage, pp. 649-688. (in German)

Gooch, S., Ashbrook, K., Taylor, A. \& Székely, T. 2015. Using dietary analysis and habitat selection to inform conservation management of reintroduced Great Bustards Otis tarda in an agricultural landscape. - Bird Study 62(3): 289-302. DOI: $10.1080 / 00063657.2015 .1050993$

Greschik, J. 1939. Vérkilövellő bogarak madaraink táplálékában - Blutausspritzende Käfer in der Nahrung unserer Vögel [Blood-spattering beetles in the diet of our birds]. - Aquila 42-45: 613-634. (in Hungarian with German Summary)

Hellmich, J. 1995. Grosstrappen essen Wassermelonen [Great Bustard eat watermelon]. - Ornithologische Mitteilungen 47(12): 309-311. (in German)

Heneberg, P. 2016. On Otis tarda and Marquis de Sade: what motivates male Great Bustards to consume Blister Beetles (Meloidae)? - Journal of Ornithology 157: 1123-1125. DOI: 10.1007/s10336-016-1369-8

Hennicke, C. R. (ed.). 1905. Der Grosstrappe - Otis tarda L. [The Great Bustard]. - Naumann Naturgeschichte der Vögel Mitteleuropas. VII. Band, pp. 57-72. (in German)

Horváth, L. 1958. Otididae - Túzokfélék [Otididae - Bustards]. - In: Székessy, V. (ed.) Aves - Madarak, Magyarország állatvilága, Fauna Hungariae XXI. 5. füzet, pp. 26-30. (in Hungarian)

Huszthy, Z. C. 1781. 39. Der Ungarische Trappe [The Hungarian Bustard]. - Ungarisches Magazin 1: 466-477. (in German)

Isakov, J. A. \& Flint, V. E. 1989. Otis tarda - Grosstrappe [Otis tarda - Great Bustard]. - In: Potapov, R. L. \& Flint, V. E. (eds.) Handbuch der Vögel der Sowjetunion Bd. 4. Galliformes, Gruiformes [Handbook of birds of Sowiet Union Bd. 4. Galliformes, Gruiformes]. - A. Ziemsen Verlag, Wittenberg, Lutherstadt, pp. 363-375. (in German)

Jourdain, F. C. R. 1948. Great Bustard - Otis tarda tarda L. - Food. - In: Witherby, H. F., Jourdain, F. C. R., Ticehurst, N. F. \& Tucker, B. W. The Handbook of British Birds, Vol. 4. - H. F. \& G. Witherby Ltd., London, pp. 438-439.

Király, G. (ed.) 2009. Új magyar füvészkönyv [The new Hungarian herbal book]. Magyarország hajtásos növényei. Határozókulcsok. - Aggteleki Nemzeti Park Igazgatóság, Jósvafő (in Hungarian)

Kistjakinskij, O. 1957. Fauna Ukraina [Fauna of Ukraine - Birds]. - Tom. 7. Ptaki, p. 128. (in Russian)

Kollar, H. P. 1996. Action plan for the Great Bustard (Otis tarda) in Europe. - In: Heredia, B., Rose, L. \& Painter, M. (eds.) Globally threatened birds in Europe. - Action plan. Council of Europe Publishing, BirdLife International, pp. 245-260.

Kollar, H. P. 2001. Aktionsplan - Schutz für di Großtrappe in Österreich [Action Plan - Conservation for the Great Bustard in Austria]. - Bundesministerium für Land- und Forstwissenschaft Umwelt und Wasserwirtschaft \& WWF Österreich, Wien (in German with English Summary)

Lane, S. J., Alonso, J. C., Alonso, J. A. \& Naveso, M. A. 1999. Seasonal changes in diet and diet selection of Great Bustards (Otis t. tarda) in north-west Spain. - Journal of Zoology 247: 201-214. DOI: 10.1111/j.1469-7998.1999. tb00984.x

Lucio, A. J. 1985. Datos sobre la alimentacion de la avutarda (Otis tarda L., 1758) en la Cuenca del Duero [Data about the feeding of the Great Bustard (Otis tarda L., 1758) in the Duero Basin]. - Alytes 3: 69-85. (in Spanish)

Mansfeld, K. 1958. Aves. - In: Sorauer, P. (ed.) Handbuch der Pflanzenkrankheiten [Handbook of plant diseases]. Bd. 5. - Paul Parey Verlag, Hamburg-Berlin (in German)

Merkl, O. \& Vig, K. 2009. Bogarak a Pannon régióban [Beetles (Coleoptera) in the Pannon Region]. - Vas megyei Múzeumok Igazgatósága, B. K. L. Kiadó \& Magyar Természettudományi Múzeum, Szombathely (in Hungarian)

Moltoni, E. 1968. Notizie sull' tarda - Otis tarda tarda L. - in Italia [News on the Great Bustard (Otis tarda tarda L.) in Italy]. - Rivista Italiana di Ornitologia 38: 223-234. (in Italian)

Morales, M. B. \& Martín, C. A. 2002. Otis tarda - Great Bustard. - BWP Update The Western Palearctic 4(3): 217232. 
Nagy, Sz. 2009. International single species action plan for the Western Palearctic population of Great Bustard, Otis tarda tarda. - BirdLife International on behalf of the European Commission

Nečas, J. \& Hanzl, R. 1956. Rozsirenie a bionomia dropa vel'keho eurosibirskeho - Otis tarda Linn. [Spreading and bionomy of Euro-Siberian Great Bustard Otis tarda Linn.]. - Sbornik Krajského Múzea v Trnave 2: 1-29. (in Slovakian)

Niethammer, G. 1942. Handbuch der Deutschen Vogelkunde, Band 3. [Handbook of German Ornithology, Vol. 3.] Tubinares, Podicipedes, Colymbi, Columbae, Pterocletes, Alcae, Laro-Limicolae, Otides, Grues, Ralli, Galli Nachträge. - Akademischer Verlagsgesellschaft, Leipzig (in German)

Palacios, F., Garzon, J. \& Castroviejo, J. 1975. La alimentacion de la Avutarda (Otis tarda) en Espaňa, especialmente en primavera [The diet of Great Bustard (Otis tarda) in Spain, especially in spring]. - Ardeola 21(1): 347-406. (in Spanish)

Palaus Soler, F. J. 1960. Notas orntológicas del noreste de España (con algunos datos de Levante) [Ornithological notes of the northeast of Spain (with some data from Levante)]. - Ardeola 6(1): 221-234. (in Spanish)

Potts, G. R. 1986. The Partridge. Pesticides, Predation and Conservation. - Harper Collins Publishers, London

Potts, G. R. 2012. Partridges. Countryside Barometer. - Harper Collins Publishers, London

Raab, R., Julius, E., Greis, L., Schütz, C., Spakovszky, P., Steindl, J. \& Schönemann, N. 2014. Endangering factors and their effect on adult Great Bustards (Otis tarda) conservation efforts in the Austrian LIFE and LIFE+ projects. - Aquila 121: 49-63.

Raab, R., Schütz, C., Spakovszky, P., Julius, E. \& Schulze, C. H. 2015. Optimising the attractiveness of winter oilseed rape fields as foraging habitat for the West Pannonian Great Bustard Otis tarda population during winter. - Bird Conservation International 25: 366-376.

Redondo, A. \& Tortosa, F. S. 1994. Status and habitat use of a population of Great Bustard in Southern Spain. - Avocetta 18: 81-84.

Rjabov, V. F. \& Ivanova, Z. J. 1971. K ekologii drofü v Szevernom Kazahsztane [To the ecology of Great Bustard in Northern Kazakhstan]. - Vestnik Moskowskogo Univ. 5: 23-31. (in Russian)

Rocha, P., Marques, A. T. \& Moreira, F. 2005. Seasonal variation in Great Bustard Otis tarda diet in South Portugal with a focus on the animal component. - Ardeola 52(2): 371-376.

Rörig, G. 1900. Magenuntersuchungen land- und forstwirtschaftlich wichtiger Vögel [Stomachs analysis of agricultural and forestry important birds]. - Arb. a. d. Biol. Abtlg. f. Land- und Forstwirtschaft am Kaiserl. Gesundheitsamte (in German)

Sánchez-Barbudo, I. S., Camarero, P. R., García-Montijano, M. \& Mateo, R. 2012. Possible cantharidin poisoning of a Great Bustard (Otis tarda). - Toxicon 59: 100-103. DOI: 10.1016/j.toxicon.2011.10.002

Spakovszky, P., Pellinger, A. \& Burda, B. 2011. A mosoni túzok (Otis tarda) állomány hosszú távú fenntartásának természetvédelmi problémái [Nature conservation problems of sustenaining the population of the Great Bustard (Otis tarda) on the Moson Plain on the long term]. - Ornis Hungarica 19(1-2): 133-140. (Hungarian with English Summary)

Spangenberg, W. 1951. Drofa [Great Bustard]. - In: Dementiew, G. P. (ed.) Ptici Sovjetszkogo Szojuza, Vol. 2. [Birds of Sowiet Union, Vol. 2.]. - Moskwa, pp. 139-168. (in Russian)

Stegman, B. 1955. Endemizm v avifauna verhaziszkih sztyepej [Endemizms in the avifauna of Northasian steppe].Pomjati Akademika L. Sz. Berga. Moskwa (in Russian)

Sterbetz, I. 1971. A hansági túzokállomány természetvédelmi problémái [The nature conservation problems of the Great Bustard population in Hanság]. - Arrabona 13: 99-108. (in Hungarian with German Summary)

Sterbetz, I. 1977. A túzok (Otis tarda L.) környezete Magyarországon [The environment of Great Bustard (Otis tar$d a$ ) in Hungary]. - Aquila 83: 53-73. (in Hungarian with English Summary)

Suárez, F. S. 2002. La alimentación de la avutarda en la provincia de Badajoz [Alimentation of Great Bustard in the pérovince of Badajoz]. - Quercus 194: 14-17. (in Spanish)

Thaisz, L. 1899. A növényekkel táplálkozó madarak hasznos vagy káros voltának elbírálása begytartalmak elemzése alapján. - Kritische Bestimmung der Nützlichkeit oder Schädlichkeit der pflanzenfressenden Vögel auf Grund des Kropfinhaltes [Judgement the useful or harmful effects of birds feeding on plants by analyzing the content of stomachs]. - Aquila 6: 133-168. (in Hungarian and German)

Vadász, Cs. \& Lóránt, M. 2014. Key mortality causes of the Great Bustard (Otis tarda) in Central Hungary: an analysis of known fatalities. - Ornis Hungarica 22: 32-41. DOI: 10.2478/orhu-2014-0016

Vasvári, M. 1942. A nünüke, mint madártáplálék - Maiwürmer als Vogelnahrung [Blister Beetle as a bird feed]. Aquila 46-49: 475-476., 506. (in Hungarian and German) 